\title{
Genomic diversity dynamics in conserved chicken populations are revealed by genome-wide SNPs
}

\author{
Mengmeng Zhang ${ }^{1 \dagger}$, Wei Han ${ }^{2 \dagger}$, Hui Tang ${ }^{3 \dagger}$, Guohui Li ${ }^{2}$, Minjie Zhang ${ }^{1,5}$, Ran Xu', Yijun Liu ${ }^{1,6}$, Tao Yang ${ }^{1}$,
} Wenting $\mathrm{Li}^{1,4^{*}}$, Jianmin Zou ${ }^{2^{*}}$ and Keliang $\mathrm{Wu}^{1 *}$ (D)

\begin{abstract}
Background: Maintaining maximum genetic diversity and preserving breed viability in conserved populations necessitates the rigorous evaluation of conservation schemes. Three chicken breeds (Baier Yellow Chicken (BEC), Beijing You Chicken (BYC) and Langshan Chicken (LSC)) are currently in conservation programs in China. Changes in genetic diversity were measured by heterozygosity, genomic inbreeding coefficients, and autozygosity, using estimates derived from runs of homozygosity $(\mathrm{ROH})$ that were identified using SNPs.

Results: Ninety DNA samples were collected from three generations for each breed. In the most recent generation, the highest genetic diversity was observed in LSC, followed by BEC and BYC. Inbreeding coefficients based on ROH for the three breeds declined slightly between the first and middle generations, and then rapidly increased. No inbreeding coefficients exceeded 0.1. Population structure assessments using neighbor-joining tree analysis, principal components analysis, and STRUCTURE analysis indicated that no genetic differentiation existed within breeds. LD decay and $\mathrm{ROH}$ at different cut-off lengths were used to identify traces left by recent or ancient inbreeding. Few long $\mathrm{ROH}$ were identified, indicating that inbreeding has been largely avoided with the current conservation strategy. The observed losses in genetic diversity and occurrences of inbreeding might be consequences of sub-optimal effective population sizes.

Conclusions: The conserved Chinese chicken populations have high genomic diversity under the current conservation program (R: F). Furthermore, this study highlights the need to monitor dynamic changes in genetic diversity in conserved breeds over successive generations. Our research provides new insights into genetic diversity dynamics in conserved populations, and lays a solid foundation for improving conservation schemes.
\end{abstract}

Keywords: Chinese indigenous chicken, Genomic diversity, Conservation scheme, Genome-wide SNPs

\section{Background}

As one of the earliest centers of domestication for chickens, China has the most abundant chicken genetic resources in the world, with 107 indigenous chicken breeds. These breeds play an essential role in the Chinese poultry

\footnotetext{
*Correspondence: liwenting_5959@hotmail.com; jaszjm@163.com; liangkwu@cau.edu.cn

${ }^{\dagger}$ Mengmeng Zhang, Wei Han and Hui Tang contributed equally to this work. 'Department of Animal Genetics and Breeding, National Engineering Laboratory for Animal Breeding, College of Animal Science and Technology, China Agricultural University, Beijing 100193, People's Republic of China ${ }^{2}$ National Chickens Genetic Resources, Institute of Poultry Science, Chinese Academy of Agricultural Science, Yangzhou 225125, People's Republic of China

Full list of author information is available at the end of the article
}

industry due to the popularity of traditional cuisine. Because these chickens typically exhibit high adaptability to variable environments, strong disease-resistance, and produce high-quality meat and eggs, they are an important breeding resource to meet future market demands [1]. However, a sizeable fraction of indigenous chicken breeds (21.3\% in the world) are under threat of extinction because breeding populations are too small for genetic sustainability [2]. Twenty-three Chinese indigenous chicken breeds have been listed in the national conservation catalogue and are currently managed under what is thought to be an optimal conservation scheme. However,

(c) The Author(s). 2018 Open Access This article is distributed under the terms of the Creative Commons Attribution 4.0 International License (http://creativecommons.org/licenses/by/4.0/), which permits unrestricted use, distribution, and 
the effectiveness of the scheme has never been evaluated over the long term.

An efficient in situ conservation scheme relies on an effective population size, as well as an effective selection and mating strategy [1]. The recommended effective population size is 50 , which is not only sufficient to maintain population fitness, but is also small enough to be monitored and managed easily $[1,3]$. The principle of a selection and mating strategy is to minimize the average kinship between selected parents [1]. The mating systems that are in use are: (i) random mating and random selection (R: R), (ii) random mating within families, with one son kept per sire family and one daughter kept per dam family (R: F), and (iii) family rotational mating (F: R) [4].

Results from a study that compared the effectiveness of these three mating systems suggest that $\mathrm{F}: \mathrm{R}$ can sustain $90 \%$ of genetic diversity in a livestock population for more than 100 years [4]. Other studies demonstrate that F: R can reduce inbreeding in populations $[5,6]$, however, implementation of the F: R scheme requires substantial effort. The contemporary conservation scheme in China relies mainly on the R: F mating system. Although simulation experiments suggest that R: F and F: R perform similarly in maintaining genetic diversity in a conserved population [4], few empirical studies have evaluated their effectiveness.

The genetic diversity dynamics in successive generations within a conserved population directly reflect the effectiveness of a conservation scheme. DNA markers can be used efficiently to estimate the genetic diversity within and between conserved populations [7]. Various DNA marker systems have been used to assess chicken genetic diversity, such as RAPDs [8], AFLPs [9], and microsatellites [10-12]. SNPs, which are densely distributed across genomes, have also been used to estimate genetic diversity and population structure with high accuracy $[13,14]$.

In order to investigate the effectiveness of the schemes that are currently used to conserve chicken genetic resources in China, we evaluated the genetic diversity of three conserved indigenous chicken breeds. SNPs, identified using high-throughput DNA sequencing in samples obtained from three generations per breed, were used to estimate diversity and track changes across time. These data can be used to evaluate and improve ongoing conservation efforts.

\section{Methods}

\section{Ethics statement}

Sample collection procedures strictly followed protocols approved by the Animal Welfare Committee of China Agricultural University (Approval Number: XK257).

\section{Sampling}

Three Chinese indigenous chicken breeds that have been enrolled in conservation programs were used in this study: Baier Yellow Chicken (BEC), Beijing You Chicken (BYC), and Langshan Chicken (LSC). Different geographical and environmental factors have contributed to the unique characteristics of these breeds. The Baier Yellow chicken, mainly produced in Jiangxi Province, has a distinct appearance with white ears and yellow feathers, beak, and shanks. It is a rare prematurity and egg-type breed. The Langshan chicken is a classic dual-purpose breed that originated in Jiangsu Province. This is one of the oldest breeds and is unusually tall, with long legs and a tail carried at a high angle. The Beijing You chicken is an ancient breed that originated during the Qing Dynasty in Beijing. Known as "royal chickens", they are valued for their high-quality meat and eggs, and are uniquely marked by a crest on the head, a beard under the lower jaw, and feathers on both shanks [15-17].

These breeds have been managed for optimal conservation as part of the National Chicken Genetics Resources program (Jiangsu, NCGR). Briefly, the conservation goals are that population sizes should be kept constant across generations (30 males and 300 females), and random mating should be enforced within families, with one son kept per sire family and one daughter kept per dam family ( $R$ : F). Samples from 270 individuals were collected from NCGR, with three generations per breed and 30 individuals per generation. Samples are identified by breed abbreviation and the last two digits of the year in which samples were obtained (e.g., samples collected from BEC in 2007 are designated BEC07). Breed and sampling information is summarized in Table 1. Blood samples were collected from the wing vein and stored at $-20^{\circ} \mathrm{C}$. Genomic DNA was extracted following the protocol accompanying the DNeasy Blood \& Tissue Kit (Qiagen Inc., Valencia, California, USA). $3 \mu \mathrm{g}$ high quality DNA was used to construct sequencing libraries for each sample.

\section{Genotyping and data preparation}

All DNA samples were subjected to genotyping by sequencing (GBS) using an Illumina HiSeq 4000 sequencer (Illumina, San Diego, CA, USA) after double enzyme digestion (MseI and HaeIII). The initial data set was filtered to exclude low-quality reads, and then aligned to the chicken genome (version: Gallus_gallus 4.0) using BWA (v0.7.8) [18]. PCR duplicates were removed using SAMtools rmdup (v0.1.19) [19]. Sequencing variants were identified using SAMtools mpileup (v0.1.19, arguments: -q 1 -C 50 -S -D -m 2 -F 0.002) and BCFtools view (arguments: -Q 20 -d 1 -D 8000). Variants satisfying all of the following criteria were retained for further analysis: coverage depth $\geq 1$ and $\leq 8000$, RMS mapping quality $>20$, and distance between adjacent SNPs $\geq 5 \mathrm{bp}$. Variants were annotated using ANNOVAR with default parameters [20].

Quality control procedures were implemented using PLINK 1.90 [21]. SNPs were required to meet the following 
Table 1 Samples obtained from conserved populations of three indigenous chicken breeds

\begin{tabular}{|c|c|c|c|c|c|c|c|c|c|}
\hline \multirow[t]{2}{*}{ Breed } & \multirow[t]{2}{*}{ Abbr. } & \multirow[t]{2}{*}{ Conservation initial year } & \multicolumn{2}{|c|}{ Population size } & \multirow[t]{2}{*}{ Geographic location } & \multirow{2}{*}{$\begin{array}{l}\text { Production } \\
\text { type }\end{array}$} & \multicolumn{2}{|c|}{ Samples $(n)$} & \multirow[t]{2}{*}{ Years } \\
\hline & & & Sire & Dam & & & Sire & Dam & \\
\hline \multirow[t]{3}{*}{ Baier Yellow Chicken } & $\mathrm{BEC}$ & 1998 & 30 & 300 & Jiangxi & Egg type & 10 & 20 & 2007 \\
\hline & & & 30 & 300 & & & 10 & 20 & 2010 \\
\hline & & & 30 & 300 & & & 10 & 20 & 2015 \\
\hline \multirow[t]{3}{*}{ Beijing You Chicken } & BYC & 1976 & 30 & 300 & Beijing & Dual purpose & 10 & 20 & 2007 \\
\hline & & & 30 & 300 & & & 10 & 20 & 2010 \\
\hline & & & 30 & 300 & & & 10 & 20 & 2015 \\
\hline \multirow[t]{3}{*}{ Langshan Chicken } & LSC & 1998 & 30 & 300 & Jiangsu & Dual purpose & 10 & 20 & 2010 \\
\hline & & & 30 & 300 & & & 10 & 20 & 2012 \\
\hline & & & 30 & 300 & & & 10 & 20 & 2015 \\
\hline
\end{tabular}

criteria: call rate $\geq 95 \%$, minor allele frequency $(\mathrm{MAF}) \geq$ 0.05 , missing rate $\leq 0.01$, and Hardy-Weinberg equilibrium test $P$-value $>10 \mathrm{e}-6$.

\section{Preparation of data prior to calculation of genetic diversity}

Before genetic diversity was estimated, linkage disequilibrium (LD) "pruning" was conducted using PLINK (v1.90, arguments: --indep-pairwise 505 0.2).

Nine generation-based sample pools (BEC07, BEC10, BEC15; BYC07, BYC10, BEC13; LSC10, LSC12, LSC15) were used to calculate genetic diversity, as reflected by expected heterozygosity $(\mathrm{He})$, observed heterozygosity $(H o)$, proportion of polymorphic markers $\left(P_{N}\right)$, and allelic richness $\left(A_{R}\right)$. He, $\mathrm{Ho}$ and $P_{N}$ were calculated using PLINK 1.90 with the default settings. $A_{R}$ estimates were determined using ADZE v1.0 [22].

\section{Inbreeding coefficient}

Two measures of inbreeding coefficient were calculated for each chicken population.

Inbreeding coefficient based on the mating plan $\left(F_{\mathrm{ES}}\right)$ : The estimation of effective population size $(\mathrm{Ne})$ was based on number of sires and dams, following Wright's model [23]. Computation of $\mathrm{Ne}$ requires the numbers of males $\left(N_{\mathrm{m}}\right)$ and females $\left(\mathrm{N}_{\mathrm{f}}\right)$ in each population that participated in the R: F program, and is calculated using the equation: $N e=\frac{3 N f+N m}{16 N m N f}$. The increment of hypothetical inbreeding $(\Delta F)$ was calculated using the equation: $\Delta F=\frac{1}{2 N e}$.

Inbreeding coefficient based on runs of homozygosity $\left(F_{\mathrm{ROH}}\right)$ : A run of homozygosity is defined as a region $>100 \mathrm{~Kb}$ containing $>50$ SNPs. $F_{\mathrm{ROH}}$ was calculated using PLINK v1.90 (with parameters --file BEC07_qc --ibc --allow-extra-chr --chr-set 28 --out BEC07) and is the fraction of the genome spanned by runs of homozygosity [24].

\section{Analysis of population structure}

To reduce noise due to linkage disequilibrium, SNPs with a pair-wise genotype $r^{2}$ value $\geq 0.2$ were removed from the data set. A principal component analysis (PCA) [25] was conducted using PLINK and visualized with the SNPRelate R package [26]. A neighbor-joining (NJ) tree was constructed with Nei's genetic distances [27] using the phylogeny program MEGA v7.0 [28] and displayed with FigTree v1.4.3 [29]. The genetic structures of the 9 sub-populations described above were analyzed with STRUCTURE v2.3.4 [30], using admixture and a correlated allele model $[30,31]$. Ten independent runs were performed with $\mathrm{K}$ ranging from 1 to 10 , with a burn-in period length of 10,000, followed by 100,000 Markov chain Monte Carlo (MCMC) repetitions, and 20 replications for each $\mathrm{K}$ value. STRUCTURE HARVESTER [32] was utilized to determine the optimal $K$ value by comparing the likelihood of the data ( $\mathrm{LnK}$ ) for different values of $\mathrm{K}[\ln \mathrm{P}(\mathrm{X} \mid \mathrm{K})]$ and by examining the second-order rate change of $\ln \mathrm{P}(\mathrm{X} \mid \mathrm{K}), \Delta \mathrm{K}[33,34]$. Results for $\mathrm{K}=2$ to $\mathrm{K}=9$ are included in this report.

\section{Estimation of genetic differentiation}

The unbiased genetic differentiation estimate, $\mathrm{F}_{\mathrm{ST}}$ [35], was calculated using VCFtools v0.1.14 [36] with the quality-controlled SNP dataset to estimate genetic differentiation between populations (with parameters --vcf chicken_qc.vcf --weir-fst-pop BEC.txt --weir-fst-pop NLS.txt --out BEC_NLS).

\section{Estimation of nucleotide diversity}

Genome-wide nucleotide diversity $(\pi)$ was computed for each breed using VCFtools v0.1.14 [36] (parameters --vcf BEC_qc.recode.vcf --window-pi 100,000 --window-pi-step 10,000 --out BEC).

\section{Linkage disequilibrium decay}

LD was evaluated as the correlation coefficient $\left(\mathrm{r}^{2}\right)$ between alleles at two separate SNP loci [37]. Within each 
population, all pairs of autosomal SNPs with MAF $>0.05$ and Hardy-Weinberg equilibrium $P$-value $>10 \mathrm{E}-6$ were used to calculate $\mathrm{r}^{2}$ with Haploview [38]. Inter-SNP distances from $0 \mathrm{~kb}$ to $500 \mathrm{~kb}$ were consolidated into 5 bins.

\section{Effective population size}

Effective population size $(\mathrm{Ne})$ was estimated according to the random mating model of linkage disequilibrium, using default parameters in $\mathrm{N}_{\mathrm{E}}$ ESTIMATOR v2.01 [39]. $\mathrm{Ne}$ estimates for each breed were calculated as the average of the estimates for macrochromosomes (gga1-gga5) [40] (Axelsson et al., 2005).

\section{Runs of homozygosity}

Runs of homozygosity (ROH) were identified for each of the 9 sub-populations using PLINK v1.90. The ROH program slides a moving window of $1 \mathrm{Mb}$ across the genome to estimate homozygosity. One heterozygous and five missing calls per window were allowed to avoid false negatives caused by occasional genotyping errors or missing genotypes. The minimum length and SNP counts required for each $\mathrm{ROH}$ were $100 \mathrm{~kb}$ and 50 SNPs, respectively. Additional statistical significant tests were conducted to detect the differences in genome-wide homozygosity levels among populations with three measures (NSEG, KB, KBAVG).

\section{Results}

\section{Descriptive statistics}

To assess genetic diversity, DNA samples from three indigenous chicken breeds (BEC, BYC, and LSC) were subjected to high-throughput DNA sequencing. Two hundred seventy individuals, representing three generations per breed and 30 individuals per generation, yielded $120 \mathrm{~Gb}$ of high-quality sequence data. About $99.7 \%$ of the reads mapped to the reference genome (Gallus_gallus 4.0) for each individual, providing $\sim 8 \mathrm{X}$ average genome coverage. 6,950,965 SNPs were identified in the initial screen. $6,234,592$ SNPs were excluded because they deviated from Hardy-Weinberg equilibrium (1,244,248 SNPs), exhibited minor allele frequency $\leq 0.05(4,959,232 \mathrm{SNPs})$, or were located on non-autosomal or small chromosomes $(31,112$ SNPs). 716,373 SNPs met criteria for inclusion in the final data set. The average physical distance between neighboring SNPs was $1.34 \mathrm{~kb}$, ranging from $1.10 \mathrm{~kb}$ on GGA6 to $5.15 \mathrm{~kb}$ on GGA25 (Additional file 1: Table S1). The distribution of SNPs across all chromosomes is shown in Additional file 2: Figure S1.

\section{Genetic diversity within the BEC, BYC and LSC breeds}

All three breeds maintained relatively high genetic diversity in the R: F conservation scheme (see scheme definitions in Materials and Methods). LSC exhibited the highest genetic diversity as measured by $H o$ (0.2348), $H e(0.2250), A_{R}$ (1.235), and $P_{N}(0.8130)$ (Table 2). An analysis across three
Table 2 Genetic diversity measurements for nine subpopulations from three chicken breeds

\begin{tabular}{lllllll}
\hline Population & Ho & He & $P_{N}(\%)$ & $A_{R}$ & $F_{E S}{ }^{a}$ & $F_{R O H}{ }^{b}$ \\
\hline BEC07 & 0.2250 & 0.2195 & 82.41 & 1.225 & 0.0567 & 0.0500 \\
BEC10 & 0.2281 & 0.2208 & 81.64 & 1.228 & 0.0748 & 0.0481 \\
BEC15 & 0.2211 & 0.2073 & 76.06 & 1.221 & 0.1043 & 0.0553 \\
BEC average & 0.2247 & 0.2159 & 80.04 & 1.225 & 0.0789 & 0.0511 \\
BYC07 & 0.2223 & 0.2173 & 80.95 & 1.222 & 0.1820 & 0.0698 \\
BYC10 & 0.2189 & 0.2091 & 79.11 & 1.219 & 0.1978 & 0.0613 \\
BYC15 & 0.2095 & 0.2091 & 75.83 & 1.210 & 0.2233 & 0.0925 \\
BYC average & 0.2169 & 0.2118 & 78.63 & 1.217 & 0.2010 & 0.0745 \\
LSC10 & 0.2290 & 0.2197 & 80.55 & 1.229 & 0.0748 & 0.0474 \\
LSC12 & 0.2376 & 0.2273 & 81.70 & 1.238 & 0.0867 & 0.0461 \\
LSC15 & 0.2379 & 0.2281 & 81.66 & 1.238 & 0.1043 & 0.0606 \\
LSC average & 0.2348 & 0.2250 & 81.30 & 1.235 & 0.0886 & 0.0514 \\
\hline
\end{tabular}

$\mathrm{He}$ Expected heterozygosity, $\mathrm{Ho}$ Observed heterozygosity, $P_{N}$ Proportion of polymorphic SNPs, $A_{R}$ Allelic richness, $F_{\mathrm{ES}}{ }^{\text {a }}$, inbreeding coefficient based on pedigree; $F_{\mathrm{ROH}}{ }^{\mathrm{b}}$, inbreeding coefficient based on runs of homozygosity

generations within the same breed showed that genetic diversity was highest for LSC15 with $\mathrm{Ho}$ (0.2379), $\mathrm{He}$ (0.2281) and $A_{R}$ (1.238). The highest proportion of polymorphic markers $\left(P_{N}\right)$ was observed in BEC07 (82.41\%), while BYC15 exhibited the lowest genetic diversity. As expected, BEC and BYC showed decreasing levels of diversity from the initial generation (BEC07/BYC07) to the current generation (BEC15/BYC15). Conversely, LSC displayed increasing diversity with the implementation of a conservation program (Fig. 1). However, dynamic changes in genetic diversity within breeds were less than $10 \%$ throughout the sampled generations (Additional file 3: Figure S2).

\section{Estimation of inbreeding coefficients}

Estimated inbreeding coefficients varied between breeds and conservation methods. Average $F_{\mathrm{ES}}$ ranged from 0.0789 in BEC to 0.2010 in BYC. As expected, $F_{\mathrm{ES}}$ values across generations increased as conservation procedures were maintained. In contrast, average $F_{\mathrm{ROH}}$ tended to be lower than $F_{\mathrm{ES}}$, ranging from 0.0511 in $\mathrm{BEC}$ to 0.0745 in $\mathrm{BYC}$, and $F_{\mathrm{ROH}}$ did not exhibit the steady increase that was observed for $F_{\mathrm{ES}}$. Maximum inbreeding was observed in BYC15 $\left(F_{\mathrm{ES}}=0.2010\right.$ and $\left.F_{\mathrm{ROH}}=0.0925\right)$. Correlation between $F_{\mathrm{ES}}$ and $F_{\mathrm{ROH}}$ was strongly positive $\left(\mathrm{r}^{2}=0.76\right)$.

\section{Population structure analysis}

Population structures of the three native chicken breeds, comprising 9 conservation sub-populations, were analyzed using PCA, NJ tree, and STRUCTURE. PCA showed that the first two principal components account for $17.77 \%$ (PC1) and $15.01 \%$ (PC2) of the total variability. Individuals from the 9 sub-populations clearly group into their respective breeds (Fig. 2a). The results of the 


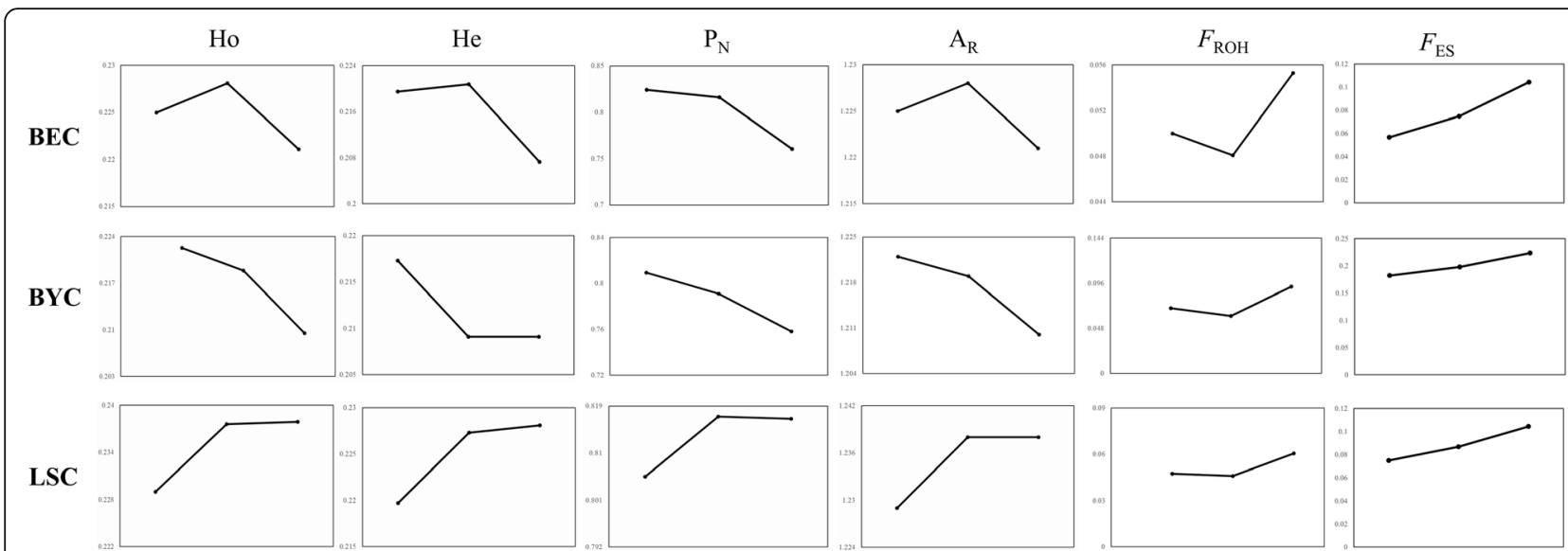

Fig. 1 Dynamic changes between different generations within breeds. Ho, observed heterozygosity; He, expected heterozygosity; $P_{N}$, proportion of polymorphic markers; $A_{R}$, allelic richness; $F_{\mathrm{ROH}}$, inbreeding coefficients based on $\mathrm{ROH}$ segments; $F_{\mathrm{ES}}$, inbreeding coefficient based on pedigree

$\mathrm{NJ}$ tree analysis were consistent with those obtained by PCA (Fig. 2b).

Figure 2c shows an admixture plot representing the 270 sampled chickens, generated using a model-based clustering approach. At a low value of $\mathrm{K}(\mathrm{K}=2)$, two distinct ancestors are apparent (BYC and LSC). BEC appears to include both LSC (as the majority component) and BYC. At $\mathrm{K}=3$, individuals cluster strongly into the three corresponding breeds, consistent with the PCA and NJ tree results (as shown in Fig. 2a and b). All generations within each breed show the same pattern. The optimum population structure inferred using the admixture model in STRUCTURE was subdivided into three sub-populations based on both $\operatorname{LnP}(\mathrm{D})$ and Evanno's $\Delta \mathrm{K}$ method (K = 3; Fig. 3). At $K=4$, BEC splits into its two main ancestors. For $\mathrm{K}=5$ to 8 , LSC appears to include two or more distinct ancestors, but at $K=9$, it groups again into one common ancestor. Finally, the, BYC breed always exhibits homogeneity, except for $\mathrm{K}=9$.

\section{Population differentiation analysis}

To investigate the extent of population differentiation between different generations within breeds and between breeds, $\mathrm{F}_{\mathrm{ST}}$ values were calculated using the filtered genotype data (Additional file 4: Table S2). The $\mathrm{F}_{\mathrm{ST}}$ values for all pair-wise population comparisons are shown in Fig. 4a. For the entire population, $\mathrm{F}_{\mathrm{ST}}$ values varied from 0.0046 to 0.1530 , and $F_{\mathrm{ST}}$ values between breeds ranged from 0.1127 to 0.1243 (Fig. 4b). $\mathrm{F}_{\mathrm{ST}}$ values are expected to be significantly higher between breeds than between generations within a breed. All $\mathrm{F}_{\mathrm{ST}}$ values between generations within a breed were below 0.05 (from 0.0046 to 0.0423 ), indicating that no obvious genetic differentiation appeared within any breed (Fig. 4a). However, these $\mathrm{F}_{\mathrm{ST}}$ values increased during conservation. For example, for $\mathrm{BEC}$, the $\mathrm{F}_{\mathrm{ST}}$ value was 0.0046 between BEC07 and BEC10, and increased to
0.0329 between $\mathrm{BEC} 07$ and BEC15. Similar trends were observed between $\mathrm{BEC} 07$ vs BEC15 (0.0329) and BEC10 vs BEC15 (0.0285).

\section{Linkage disequilibrium decay (LD decay) and effective population size}

LD for each population was estimated as the physical genomic distance at which the genotypic association $\left(\mathrm{r}^{2}\right)$ decays to less than half of its maximum value. Short-range LD was always observed in each of the three different generations within same breed (Fig. 5). As expected, LD values tended to increase as conservation continued. For example, LD values for LSC10, 12 and 15 were $13.19 \mathrm{~kb}, 16.99 \mathrm{~kb}$, and $20.10 \mathrm{~kb}$, respectively. The BEC pattern was similar: $11.56 \mathrm{~kb}$ (BEC07), $13.55 \mathrm{~kb}$ (BEC10), and $20.77 \mathrm{~kb}$ (BEC15). The highest LD value in BYC occurred in the last generation (BYC15, $24.61 \mathrm{~kb})$, but BYC07 (23.99 kb) exceeded BYC10 (17.95 kb).

Effective population size $(\mathrm{Ne})$ was estimated for autosomal chromosomes gga1 through gga28 based on linkage disequilibrium (Table 3). Average Ne differed amongst the 9 sub-populations (from 19.33 to 34.85). Within macrochromosomes (gga1-5), BEC07 had the highest estimated $\mathrm{Ne}$ (81.52) within a breed, and $\mathrm{Ne}$ declined in BEC as conservation continued. In contrast, $\mathrm{Ne}$ was lower (55.36) in LSC12 than in LSC10 (70.28) or LSC15 (73.74). Ne estimates for BYC fluctuated, with a high value (75.04) in BYC10 and lower values in BYC07 and BYC15.

\section{Runs of homozygosity (ROH)}

Runs of homozygosity ( $\mathrm{ROH}$ ) were identified in the genomes of the 9 sub-populations from all three breeds (Table 4). A genome-wide survey for autozygosity was conducted to identify regions with signatures that reflect ancient or recent inbreeding effects. We estimated $F_{\mathrm{ROH}}$, and found that the maximum values occurred in the 

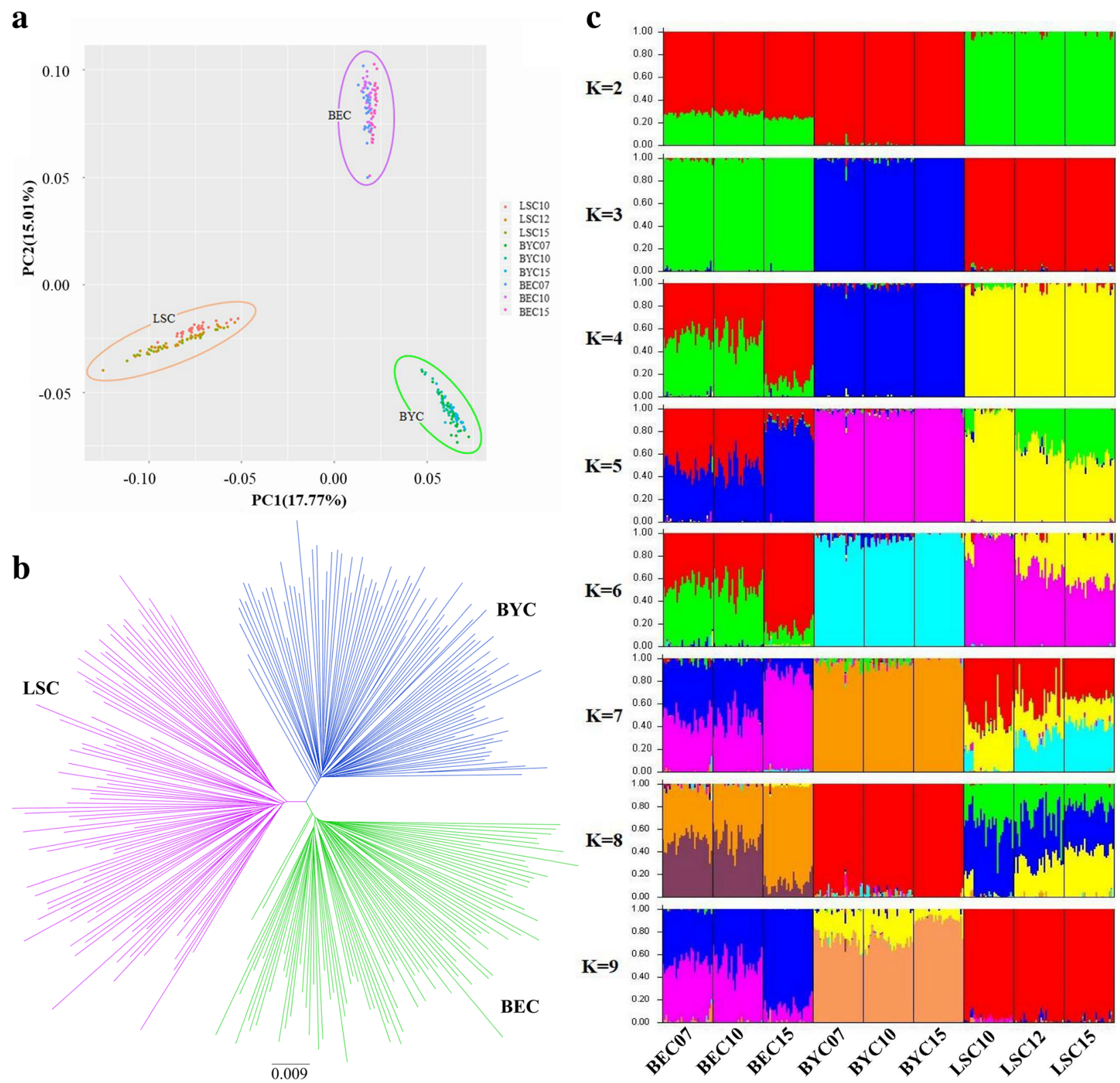

Fig. 2 a Population structures of conserved populations revealed by principal component analysis. b Neighbor-joining tree constructed using genetic sharing distances. c Admixture plot for breeds analyzed based on different number of assumed ancestors (K). BEC, Baier Yellow Chicken; BYC, Beijing You Chicken; LSC, Langshan Chicken

BYC breed. All three BYC generations exceeded 0.05 (Table 2). BYC15 had the highest level of inbreeding (0.0925), while the BEC and LSC breeds had similar and lower inbreeding levels ( 0.05).

$\mathrm{ROH}$ was then assessed to determine whether any populations exhibited evidence of recent inbreeding. All three generations in BYC had higher $\mathrm{ROH}$ values, suggesting that recent inbreeding had occurred in this breed (Table 4 and Fig. 6). Consistent with the LD decay analysis, the highest $\mathrm{ROH}$ was observed in BYC15 $\left(\mathrm{r}_{0.1248}^{2}=24.61 \mathrm{~kb}\right)$, followed by BYC07 and BYC10. We speculate that BYC inbreeding might reflect the small effective population size of this breed (Table 3). The ROH values for BEC were highest in BEC15, followed by BEC07 and BEC10, which parallels the trend observed for LD decay and $F_{\mathrm{ROH}}$. Similar patterns were observed for LSC. Homozygosity was also measured between the individuals in each sub-population using three methods (NSEG, KB, and $\mathrm{KB}_{\mathrm{AVER}}$ ) (Additional file 5: Table S3). All three measures varied significantly between BYC generations $(P<0.01)$. NSEG and KB also showed significant differences $(P<0.0001)$ between generations in BEC and NLS. 

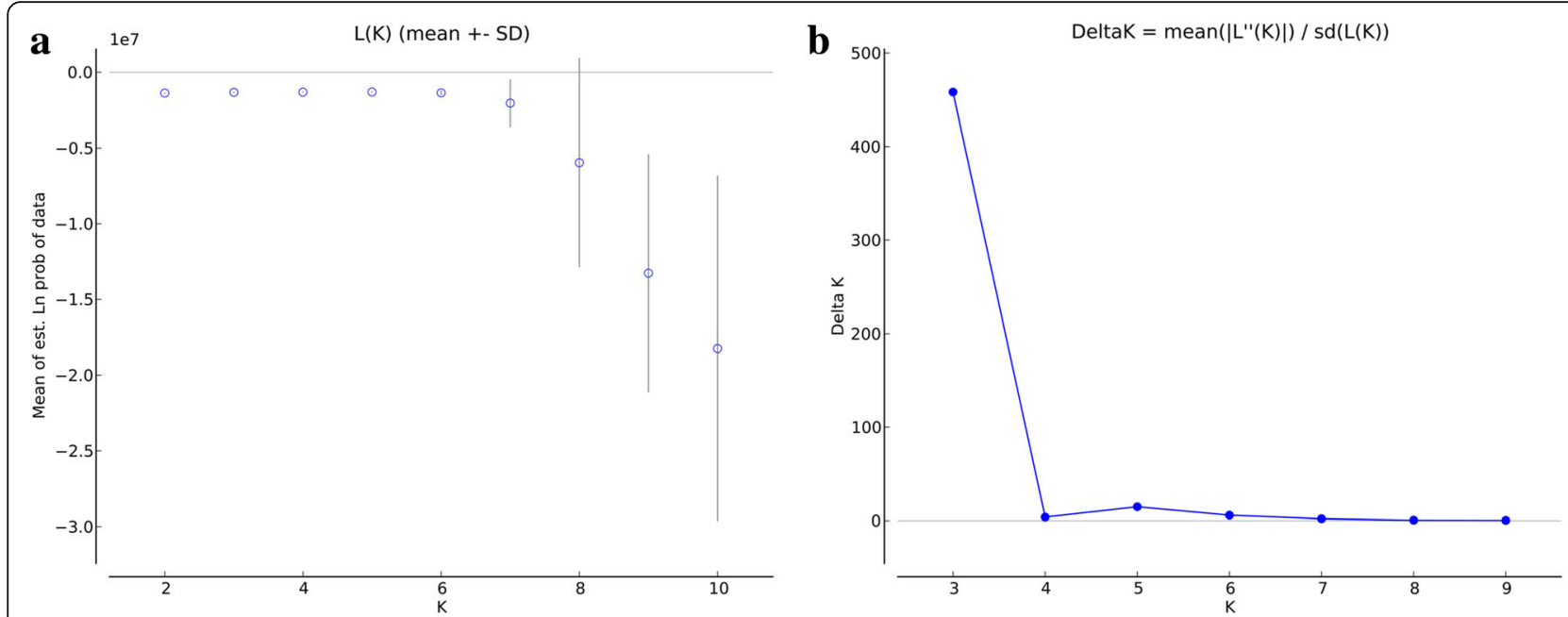

Fig. $3 L(K)$ and $\Delta K$ values using different values of $K$, as calculated by STRUCTURE Harvester. a Average likelihood of runs in STRUCTURE $L(K)$ along with number of $K$ clusters. . $\mathbf{b} \Delta K$, estimator of the optimal number of clusters $(K)$

\section{Discussion}

The chicken, one of the first animals to be domesticated, has been subjected to long-term natural selection, artificial selection, and genetic drift for diverse specific traits $[41,42]$. A variety of factors accelerated the generation of phenotypic differences and genetic variability [43, 44]. However, this variability has been threatened due to ecosystem damage and commercial breeding. In China, gene pool (live) conservation and protected regions for both ex situ and in situ conservation have been established for the management of poultry genetic resources, as exemplified by the National Chicken Genetics Resources Program (Jiangsu). Because live conservation is typically implemented using small populations, it is necessary to monitor the status of each population to evaluate the effectiveness of the management strategy. In this study, we performed genotyping by sequencing (GBS) to assess the genomic diversity of different generations from three conserved breeds (Baier Yellow Chicken, Beijing You Chicken and Langshan Chicken).

The majority of studies have estimated genetic variability in Chinese indigenous chickens using microsatellites $[45,46]$ or mtDNA $[47,48]$, but genome-wide SNPs have seldomly been used. We estimated the genetic diversity in three chicken populations using SNP markers. The results showed that all three chicken breeds have maintained rich genetic diversity in terms of heterozygosity $(\mathrm{Ho}, \mathrm{He})$, proportion of polymorphic markers $\left(P_{N}\right)$, and allelic richness $\left(A_{R}\right)$, consistent with previous studies $[45,46,49-51]$. In the most recent generations

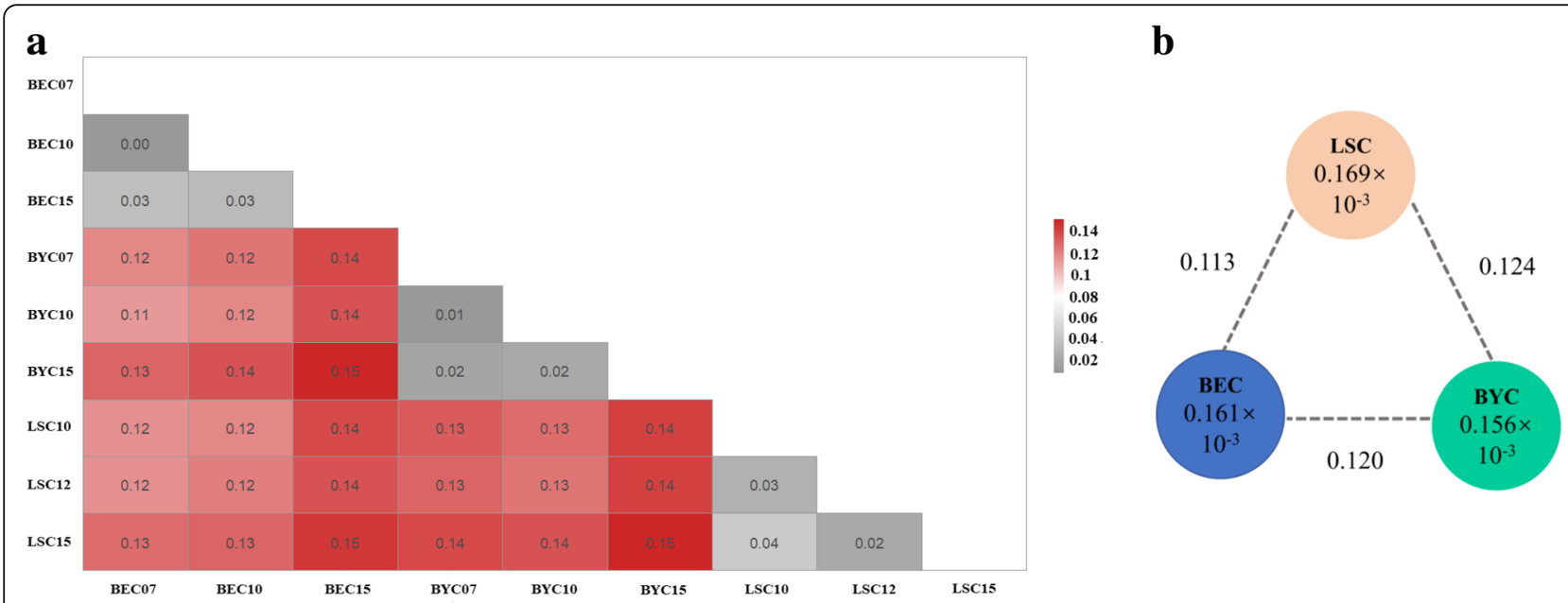

Fig. 4 a Matrix showing pairwise differentiation estimates $\left(F_{S T}\right)$ between nine breed sub-populations. b Nucleotide diversity $(\pi)$ and genetic differentiation $\left(F_{S T}\right)$ across the three breeds. The value in each circle represents a measure of nucleotide diversity for this breed, and the value on each line indicates genetic differentiation between the two breeds 


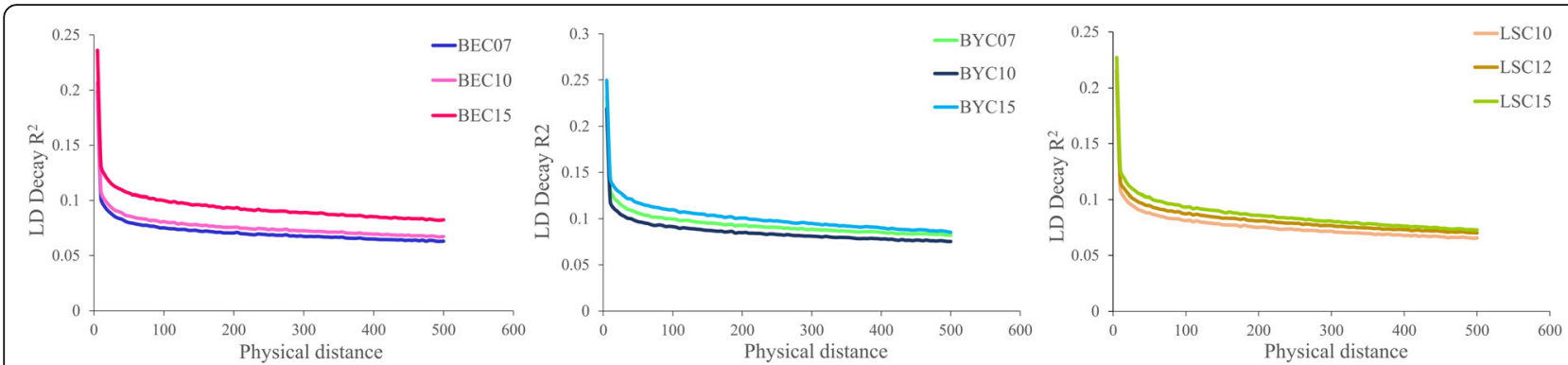

Fig. 5 Linkage disequilibrium between generations and within each breed as a function of inter-SNP distance. Physical distance is measured in $\mathrm{kb}$

Table 3 Effective population sizes (Ne) for three Chinese indigenous chicken populations

\begin{tabular}{|c|c|c|c|c|c|c|c|c|c|}
\hline \multirow{2}{*}{$\begin{array}{l}\text { Chromosome } \\
\text { number }\end{array}$} & \multicolumn{3}{|l|}{ BEC } & \multicolumn{3}{|l|}{ BYC } & \multicolumn{3}{|l|}{ LSC } \\
\hline & 2007 & 2010 & 2015 & 2007 & 2010 & 2015 & 2010 & 2012 & 2015 \\
\hline Chr1 & 108.2 & 78.3 & 56.3 & 55.7 & 101.7 & 63.8 & 86.9 & 68.4 & 102.3 \\
\hline Chr2 & 96.4 & 66.6 & 51.4 & 55.5 & 91.0 & 60.6 & 63.5 & 56.8 & 78.9 \\
\hline Chr3 & 75.6 & 69.1 & 44.4 & 52.6 & 80.9 & 58.7 & 74.7 & 50.7 & 78.6 \\
\hline Chr4 & 70.8 & 53.7 & 28.1 & 34.8 & 51.7 & 57.5 & 63.6 & 56.6 & 63.9 \\
\hline Chr5 & 56.6 & 43.3 & 27.6 & 32.9 & 49.9 & 35.8 & 62.7 & 44.3 & 45.0 \\
\hline Chr6 & 36.9 & 27.9 & 25.9 & 22.3 & 36.0 & 25.6 & 52.1 & 31.9 & 28.3 \\
\hline Chr7 & 35.0 & 30.1 & 19.3 & 21.9 & 41.3 & 35.7 & 31.1 & 27.2 & 42.2 \\
\hline Chr8 & 30.7 & 37.1 & 22.5 & 16.8 & 30.5 & 20.9 & 34.6 & 32.8 & 32.9 \\
\hline Chr9 & 37.4 & 28.9 & 13.3 & 23.8 & 28.2 & 22.2 & 27.5 & 21.3 & 28.2 \\
\hline Chr10 & 21.4 & 21.7 & 9.1 & 15.1 & 20.9 & 17.2 & 25.4 & 25.0 & 20.9 \\
\hline Chr11 & 25.6 & 21.1 & 17.9 & 17.4 & 24.1 & 16.6 & 20.7 & 22.2 & 21.9 \\
\hline Chr12 & 39.9 & 29.3 & 23.2 & 19.0 & 30.5 & 24.9 & 31.6 & 30.4 & 25.4 \\
\hline Chr13 & 19.5 & 19.7 & 16.6 & 16.5 & 23.1 & 14.8 & 22.1 & 23.5 & 21.7 \\
\hline Chr14 & 26.0 & 21.7 & 16.6 & 14.8 & 23.5 & 20.8 & 25.5 & 26.2 & 19.6 \\
\hline Chr15 & 25.3 & 24.2 & 15.2 & 11.4 & 12.5 & 8.7 & 21.5 & 29.6 & 23.7 \\
\hline Chr16 & 3.3 & 5.5 & 3.0 & 2.4 & 3.1 & 2.6 & 2.9 & 4.1 & 2.6 \\
\hline Chr17 & 29.7 & 18.7 & 12.6 & 15.1 & 22.6 & 14.8 & 21.8 & 21.3 & 22.4 \\
\hline Chr18 & 19.2 & 19.8 & 13.3 & 15.6 & 17.7 & 17.4 & 20.5 & 15.5 & 15.7 \\
\hline Chr19 & 33.3 & 25.7 & 13.1 & 15.9 & 20.4 & 18.4 & 26.2 & 18.7 & 19.0 \\
\hline Chr20 & 24.7 & 24.6 & 15.9 & 8.8 & 16.3 & 14.0 & 26.2 & 22.8 & 18.1 \\
\hline Chr21 & 27.9 & 24.1 & 11.1 & 14.5 & 16.6 & 13.0 & 18.4 & 18.0 & 25.6 \\
\hline Chr22 & 10.5 & 9.7 & 6.9 & 7.5 & 11.5 & 9.0 & 16.4 & 17.2 & 15.9 \\
\hline Chr23 & 28.3 & 18.1 & 14.3 & 16.2 & 22.7 & 17.4 & 22.3 & 22.0 & 21.9 \\
\hline Chr24 & 20.5 & 16.6 & 11.2 & 14.2 & 19.6 & 16.1 & 19.2 & 23.2 & 17.5 \\
\hline Chr25 & 13.2 & 10.0 & 9.2 & 5.8 & 8.7 & 8.9 & 18.1 & 14.7 & 14.5 \\
\hline Chr26 & 18.8 & 15.6 & 12.7 & 9.4 & 18.5 & 12.2 & 24.5 & 14.9 & 16.5 \\
\hline Chr27 & 21.7 & 22.1 & 17.0 & 15.1 & 19.6 & 9.8 & 18.6 & 21.3 & 20.0 \\
\hline Chr28 & 19.3 & 14.8 & 13.4 & 12.0 & 20.1 & 15.0 & 22.7 & 16.0 & 17.4 \\
\hline Average (Chr1-5) & 81.52 & 62.2 & 41.56 & 46.3 & 75.04 & 55.28 & 70.28 & 55.36 & 73.74 \\
\hline Average (Chr6-10) & 32.28 & 29.14 & 18.02 & 19.98 & 31.38 & 24.32 & 34.14 & 27.64 & 30.5 \\
\hline Average (Chr11-28) & 22.59 & 18.96 & 13.51 & 12.87 & 18.39 & 14.13 & 21.07 & 20.09 & 18.86 \\
\hline Average (all) & 34.85 & 28.50 & 19.33 & 20.11 & 30.83 & 23.30 & 32.19 & 27.74 & 30.74 \\
\hline
\end{tabular}


Table 4 Statistical summary for runs of homozygosity in sub-populations of three chicken breeds

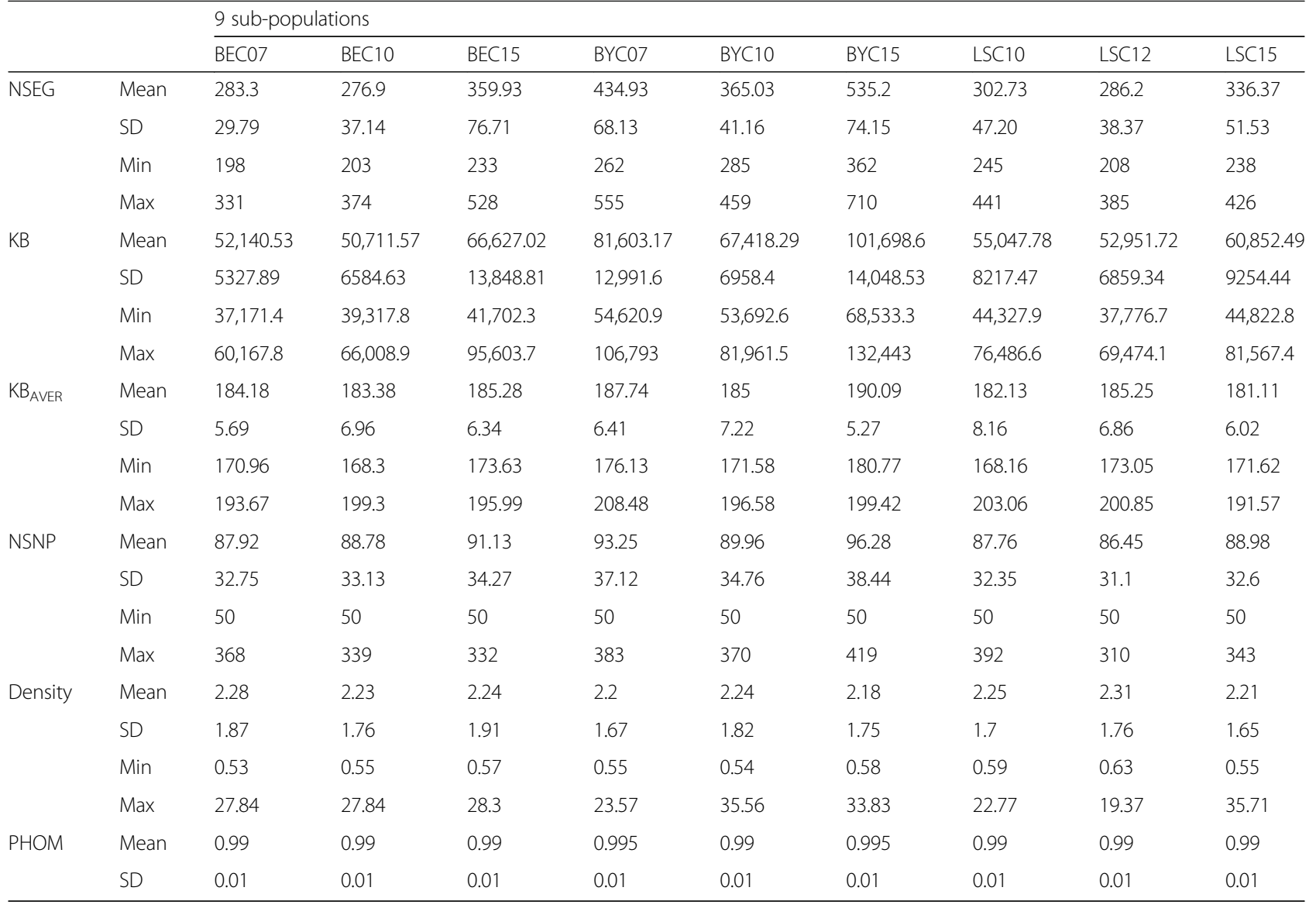

NSEG average number of segments for the individual declared homozygous

$\mathrm{KB}$ average of total number of kb contained within homozygous segments

$\mathrm{KB}_{\mathrm{AVER}}$ average size of homozygous segments

NSNP average number of SNPs in run

Density max inverse density (kb/SNP)

PHOM Proportion of sites homozygous

Min Minimum

Max Maximum

SD Standard deviation

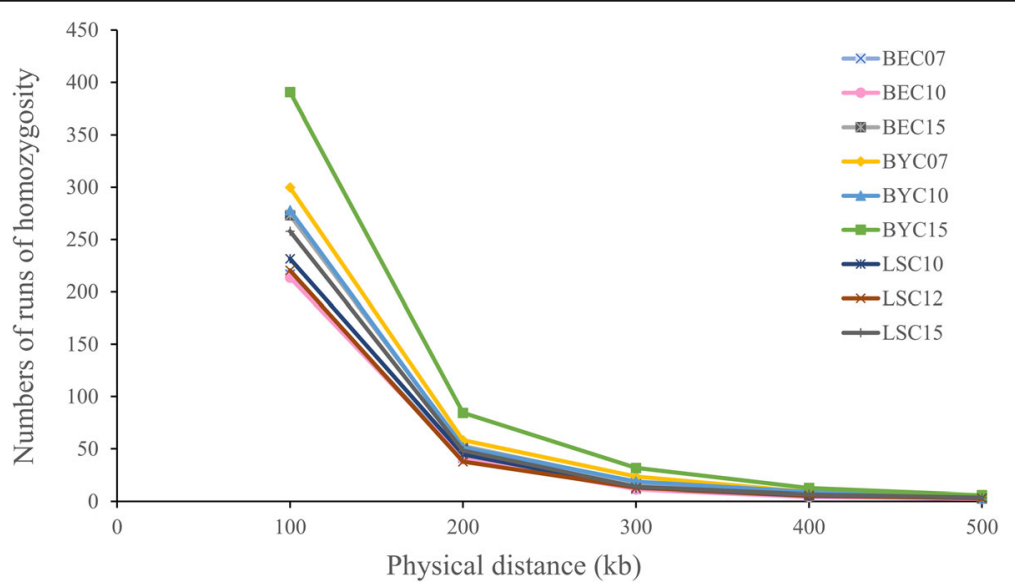

Fig. 6 Homozygosity frequency distribution derived from runs of homozygosity $(\mathrm{ROH})$ for each generation and breed 
sampled, LSC15 ranked first in genetic diversity, followed by BEC15 and BYC15 (Table 2). A study of the same populations in 2008 indicated that genetic diversity measured using microsatellites was highest in BYC, followed by LSC and BEC [45], suggesting that genetic diversity in BYC has decreased more rapidly than in the other breeds. We also observed this declining trend in the BYC breed. BYC diversity decreased from 2007 to 2015 (Table 2), while genetic variability in the BEC breed fluctuated, and LSC exhibited a slight increase. The BYC breed has been under conservation ( 39 generations) for a longer period than either LSC or BEC, which have been conserved only since 1998 ( 17 generations). The long-term practice of conservation in a small population size may reduce genetic diversity. Furthermore, all three breeds were subjected to ex situ live conservation in Jiangsu. The BYC breed, which originated in Beijing, might have adapted poorly to the environment, resulting in a loss of genetic diversity. In contrast, the LSC and BEC breeds might have adapted more easily.

The genetic diversity in all breeds changed no more than $10 \%$ between generations (Additional file 3: Figure S2). The conservation goal is to maintain $90 \%$ of the genetic diversity from the initial population and an inbreeding coefficient less than 0.1 for 100 years [52, 53]. According to our results, the genetic diversity of the three chicken populations meets conservation criteria under the current program (R: F). In particular, inbreeding events have been effectively avoided under the R: $F$ mating system, based on assessments of population structure, genetic differentiation, LD decay, and $\mathrm{ROH}$.

Nevertheless, the decline of genetic diversity should not be ignored (Fig. 1 and Table 2). The significant differences in $\mathrm{ROH}$ that we observe between generations in all three breeds also suggest that these populations have not reached the desired level of genetic stability during conservation. Both the decline in genetic diversity and the high heterozygosity across generations are indicative of genetic drift, which can be reduced by enlarging the population size. In our study, the estimated effective population sizes $(\mathrm{Ne})$, based on whole-genome SNPs for the conserved populations, were far below the required threshold of 50 individuals $[1,3]$. We also evaluated $\mathrm{Ne}$ according to chromosome size, using the classification proposed by the International Chicken Genome Sequencing Consortium [54]: large macrochromosomes (gga1-5), intermediate chromosomes (gga6-10) and micro-chromosomes (gga1128). Because micro-chromosomes have high rates of recombination, we estimated $\mathrm{Ne}$ based on the macrochromosome class (gga1-5). The maximum $\mathrm{Ne}$ was 81.52 in BEC07 and the minimum was 41.56 in BEC15 (Table 3), suggesting these conserved populations are relatively stable but also at risk. We therefore recommend that ex situ live and in situ live conservation efforts be combined to help maintain high levels of genetic diversity in the long term.

\section{Conclusions}

In summary, we collected 270 samples from three successive generations of three conserved chicken breeds. We estimated dynamic changes in genetic diversity using genome-wide SNPs, making it possible to comprehensively evaluate the current conservation scheme (R: F). The results demonstrated that the conserved Chinese chicken populations have sustained high levels of genetic variability under current conservation practices. We also compared successive generations within each breed to characterize trends in genetic diversity, allowing us to assess the effects of conservation over time. Overall, this study demonstrates an efficient strategy for assessing the success a conservation program and for improving conservation and management practices.

\section{Additional files}

Additional file 1: Table S1. The distribution of SNPS and average distances between neighboring SNPs. (DOCX $17 \mathrm{~kb}$ )

Additional file 2: Figure S1. SNP density and distribution across the genome (after quality control). (TIF $2605 \mathrm{~kb}$ )

Additional file 3: Figure S2. Fluctuations in genetic diversity among different generations within each breed. (TIF 2872 kb)

Additional file 4: Table S2. Genetic differentiation ( $F_{S T}$ values) among the 9 sub-populations in three chicken breeds. (DOCX $25 \mathrm{~kb}$ )

Additional file 5: Table S3. Differences in measures of homozygosity between individuals among 9 sub-populations in three chicken breeds. (DOCX $27 \mathrm{~kb}$ )

\section{Funding}

This study was supported by the Program for Changjiang Scholars and Innovation Research Teams in the University (IRT_15R62) and the National Basic Research Program of China (973 Program, Grant 2014CB138501).

\section{Availability of data and materials}

The sequence data generated for this project have been deposited and are available in the ENA (European Nucleotide Archive) under accession number PRJEB27583, http://www.ebi.ac.uk/ena/data/view/PRJEB27583. All relevant information supporting the results of this paper is included within the article and its additional files.

\section{Authors' contributions}

KLW conceived and designed the experiments. MMZ, WH, WTL, and HT designed and performed bioinformatics analyses and experiments. MMZ, WTL, $J M Z, G H L$, and YJL analyzed the data, and prepared the figures and tables. MMZ, WTL, MJZ, RX, and TY wrote the manuscript. All authors reviewed the manuscript. All authors read and approved the final manuscript.

\section{Ethics approval}

Chickens used in this study were collected from the National Chicken Genetics Resources program (Jiangsu, NCGR). Sample collection procedures strictly followed protocols approved by the Animal Welfare Committee of China Agricultural University (Approval Number: XK257). No wild and/or native flora were sampled.

Consent for publication Not applicable.

Competing interests

The authors declare that they have no competing interests. 


\section{Publisher's Note}

Springer Nature remains neutral with regard to jurisdictional claims in published maps and institutional affiliations.

\section{Author details Republic of China. \\ Received: 2 February 2018 Accepted: 31 July 2018 \\ Published online: 09 August 2018}

'Department of Animal Genetics and Breeding, National Engineering Laboratory for Animal Breeding, College of Animal Science and Technology, China Agricultural University, Beijing 100193, People's Republic of China. ${ }^{2}$ National Chickens Genetic Resources, Institute of Poultry Science, Chinese Academy of Agricultural Science, Yangzhou 225125, People's Republic of China. ${ }^{3}$ College of Animal Science and Technology, Shandong Agricultural University, Tai'an 271018, People's Republic of China. ${ }^{4}$ College of Animal Sciences and Veterinary Medicine, Henan Agricultural University, Zhengzhou 450002, People's Republic of China. ${ }^{5}$ Beijing Key Laboratory for Animal Genetic Improvement, Beijing 100193, People's Republic of China. ${ }^{6}$ College of Animal Science, Southwest University, Chongqing 402460, People's

\section{References}

1. Oldenbroek K. In: Oldenbroek K, editor. Utilisation and conservation of farm animal genetic resources; 2007. p. 232

2. FAO. Status and trends of animal genetic resources. Rome: Commission on Genetic Resources for Food and Agriculture; 2014.

3. Meuwissen T, Woolliams JA. Effective sizes of livestock populations to prevent a decline in fitness. Theor Appl Genet. 1994:89(7-8):1019-26.

4. Lu YF. Study on diversity of domestic animal genetic resources and conservation programs. Beijing: Vol. Ph. D. China Agricultural University; 2013

5. Honda T, Nomura T, Mukai F. Reduction of inbreeding in commercial females by rotational mating with several sire lines. Genet Sel Evol. 2004;36(5):509.

6. Windig JJ, Kaal L. An effective rotational mating scheme for inbreeding reduction in captive populations illustrated by the rare sheep breed Kempisch Heideschaap. Animal. 2008;2(12):1733-41.

7. Boettcher PJ, Tixier Boichard M, Toro MA, Simianer H, Eding H, Gandini G, Joost S, Garcia D, Colli L, Ajmone Marsan P. Objectives, criteria and methods for using molecular genetic data in priority setting for conservation of animal genetic resources. Anim Genet. 2010;41(s1):64-77.

8. Lynch M, Milligan BG. Analysis of population genetic structure with RAPD markers. Mol Ecol. 1994;3(2):91-9.

9. Schlötterer C. The evolution of molecular markers-just a matter of fashion? Nat Rev Genet. 2004;5(1):63-9.

10. Abebe AS, Mikko S, Johansson AM. Genetic diversity of five local Swedish chicken breeds detected by microsatellite markers. PLoS One. 2015;10(4): e120580.

11. Maudet C, Luikart G, Taberlet P. Genetic diversity and assignment tests among seven French cattle breeds based on microsatellite DNA analysis. J Anim Sci. 2002;80(4):942-50.

12. Pham M, Tran X, Berthouly-Salazar C, Tixier-Boichard M, Chen C, Lee Y. Monitoring of genetic diversity in Taiwan conserved chickens assessed by pedigree and molecular data. Livest Sci. 2016;184:85-91.

13. Engelsma KA: Use of SNP markers to conserve genome-wide genetic diversity in livestock; 2012.

14. Strillacci MG, Cozzi MC, Gorla E, Mosca F, Schiavini F, Román-Ponce SI, López FR, Schiavone A, Marzoni M, Cerolini S. Genomic and genetic variability of six chicken populations using single nucleotide polymorphism and copy number variants as markers. Animal. 2017;11(5):737-45.

15. Nie $\mathrm{Q}$, Ip S, Zhang X, Leung F, Yang G. New variations in intron 4 of growth hormone gene in Chinese native chickens. J Hered. 2002;93(4):277-9.

16. He C, Chen Y, Yang K, Zhai Z, Zhao W, Liu S, Ding J, Dai R, Yang L, Xu K. Genetic pattern and gene localization of polydactyly in Beijing fatty chicken. PLoS One. 2017;12(5):e0176113.

17. Chu Q, Yan Z, Zhang J, Usman T, Zhang Y, Liu H, Wang H, Geng A, Liu H. Association of SNP rs80659072 in the ZRS with polydactyly in Beijing you chickens. PLoS One. 2017:12(10):e0185953.

18. Li H, Durbin R. Fast and accurate short read alignment with burrowswheeler transform. Bioinformatics. 2009:25(14):1754-60.

19. Li H, Handsaker B, Wysoker A, Fennell T, Ruan J, Homer N, Marth G, Abecasis G, Durbin R. The sequence alignment/map format and SAMtools. Bioinformatics. 2009;25(16):2078-9.
20. Wang K, Li M, Hakonarson H. ANNOVAR: functional annotation of genetic variants from high-throughput sequencing data. Nucleic Acids Res. 2010; 38(16):e164

21. Purcell S, Neale B, Todd-Brown K, Thomas L, Ferreira MA, Bender D, Maller J, Sklar P, de Bakker PI, Daly MJ, et al. PLINK: a tool set for whole-genome association and population-based linkage analyses. Am J Hum Genet. 2007; 81(3):559-75.

22. Szpiech ZA, Jakobsson M, Rosenberg NA. ADZE: a rarefaction approach for counting alleles private to combinations of populations. Bioinformatics. 2008;24(21):2498-504.

23. Wright S. Evolution in Mendelian populations. Genetics. 1931;16(2):97-159.

24. Leutenegger A, Prum B, Génin E, Verny C, Lemainque A, Clerget-Darpoux F, Thompson EA. Estimation of the inbreeding coefficient through use of genomic data. Am J Hum Genet. 2003;73(3):516-23.

25. Price AL, Patterson NJ, Plenge RM, Weinblatt ME, Shadick NA, Reich D. Principal components analysis corrects for stratification in genome-wide association studies. Nat Genet. 2006:38(8):904-9.

26. Zheng X, Levine D, Shen J, Gogarten SM, Laurie C, Weir BS. A highperformance computing toolset for relatedness and principal component analysis of SNP data. Bioinformatics. 2012;28(24):3326-8.

27. Nei M. Genetic distance between populations. Am Nat. 1972:106(949):283-92.

28. Kumar S, Stecher G, Tamura K. MEGA7: molecular evolutionary genetics analysis version 7.0 for bigger datasets. Mol Biol Evol. 2016:33(7):1870-4.

29. Rambaut A. FigTree v1.4.0, a graphical viewer of phylogenetic trees. Edinburgh: University of Edinburgh; 2007. p. 2007.

30. Pritchard JK, Stephens M, Donnelly P. Inference of population structure using multilocus genotype data. Genetics. 2000;155(2):945-59.

31. Falush D, Stephens M, Pritchard JK. Inference of population structure using multilocus genotype data: linked loci and correlated allele frequencies. Genetics. 2003:164(4):1567-87.

32. Earl DA, VonHoldt BM. STRUCTURE HARVESTER: a website and program for visualizing STRUCTURE output and implementing the Evanno method. Conserv Genet Resour. 2012;4(2):359-61.

33. Evanno G, Regnaut S, Goudet J. Detecting the number of clusters of individuals using the software STRUCTURE: a simulation study. Mol Ecol. 2005:14(8):2611-20.

34. Janes JK, Miller JM, Dupuis JR, Malenfant RM, Gorrell JC, Cullingham Cl, Andrew RL. The K = 2 conundrum. Mol Ecol. 2017;26(14):3594-602.

35. Weir BS, Cockerham CC. Estimating F-statistics for the analysis of population structure. Evolution. 1984;6:1358-70.

36. Danecek P, Auton A, Abecasis G, Albers CA, Banks E, DePristo MA, Handsaker RE, Lunter G, Marth GT, Sherry ST. The variant call format and VCFtools. Bioinformatics. 2011;27(15):2156-8.

37. VanLiere JM, Rosenberg NA. Mathematical properties of the $r 2$ measure of linkage disequilibrium. Theor Popul Biol. 2008;74(1):130-7.

38. Barrett JC, Fry B, Maller J, Daly MJ. Haploview: analysis and visualization of LD and haplotype maps. Bioinformatics. 2005:21(2):263-5.

39. Do C, Waples RS, Peel D, Macbeth GM, Tillett BJ, Ovenden JR. NeEstimator v2 re-implementation of software for the estimation of contemporary effective population size (ne) from genetic data. Mol Ecol Resour. 2014;14(1):209-14.

40. Axelsson E, Webster MT, Smith NG, Burt DW, Ellegren H. Comparison of the chicken and Turkey genomes reveals a higher rate of nucleotide divergence on microchromosomes than macrochromosomes. Genome Res. 2005:15(1):120-5.

41. Darwin C: The variation of animals and plants under domestication, vol. 2: O. Judd; 1868.

42. Darwin C: On the Origen of species by means of natural selection: culture et civilisation; 1969.

43. Andersson L. Genetic dissection of phenotypic diversity in farm animals. Nat Rev Genet. 2001;2(2):130-8.

44. Andersson L, Georges M. Domestic-animal genomics: deciphering the genetics of complex traits. Nat Rev Genet. 2004;5(3):202-12

45. Chen G, Bao W, Shu J, Ji C, Wang M, Eding H, Muchadeyi F, Weigend S. Assessment of population structure and genetic diversity of 15 Chinese indigenous chicken breeds using microsatellite markers. Asian Australas J Anim Sci. 2008;21(3):331

46. Ou L, Li X, Xu G, Chen K, Yang H, Zhang L, Wu G, Hou Z, Xu G, Yang N. Evaluation of genetic diversity in Chinese indigenous chicken breeds using microsatellite markers. Sci China Ser C Life Sci. 2006:49(4):332-41.

47. Guo HW, Li C, Wang XN, Li ZJ, Sun GR, Li GX, Liu XJ, Kang XT, Han RL. Genetic diversity of mtDNA D-loop sequences in four native Chinese chicken breeds. Br Poult Sci. 2017;58(5):490-7. 
48. Jia XX, Lu JX, Tang XJ, Fan YF, Huang SH, Ge QL, Gao YS. Genetic diversity of Jiangsu native chicken breeds assessed with the mitochondrial DNA D-loop region. Br Poult Sci. 2018;59(1):34-9.

49. Du ZQ, Qu LJ, Li XY, Hu XX, Huang YH, Li N, Yang N. Genetic diversity in Tibetan chicken. Yi Chuan. 2004;26(2):167-71.

50. Weigend S, Hillel J, Groenen M, Tixier-Boichard M, Korol A, Kirzner V, Freidlin P, Crooijmans $R$, Vignal A, Wimmers $K$ : Assessment of biodiversity in a wide range of chicken breeds by genotyping DNA pools for microsatellite loci. 2000.

51. Weigend S, Romanov MN. Current strategies for the assessment and evaluation of genetic diversity in chicken resources. Worlds Poult Sci J. 2001;57(3):275-88.

52. Soulé M, Gilpin M, Conway W, Foose T. The millenium ark: how long a voyage, how many staterooms, how many passengers? Zoo Biol. 1986;5(2):101-13.

53. Wu CX. Poultry genetic resources in China: conservation and utilization. Beijing: XXV World's Poultry Congress; 2016. p. 88-91.

54. International Chicken Genome Sequencing Consortium. Sequence and comparative analysis of the chicken genome provide unique perspectives on vertebrate evolution. Nature. 2004:432:695.

Ready to submit your research? Choose BMC and benefit from:

- fast, convenient online submission

- thorough peer review by experienced researchers in your field

- rapid publication on acceptance

- support for research data, including large and complex data types

- gold Open Access which fosters wider collaboration and increased citations

- maximum visibility for your research: over $100 \mathrm{M}$ website views per year

At $\mathrm{BMC}$, research is always in progress.

Learn more biomedcentral.com/submissions 